Research

\title{
FAM65A as a novel prognostic biomarker in human tumors reveal by a pan-cancer analysis
}

\author{
Wenken Liang ${ }^{1,2} \cdot$ Chune $\mathrm{Mo}^{2} \cdot$ Jianfen $\mathrm{Wei}^{1} \cdot$ Wei Chen $^{3} \cdot$ Weiwei Gong ${ }^{2} \cdot$ Jianling Shi ${ }^{2} \cdot$ Xianliang Hou $^{2}$. \\ Chunhong $\mathrm{Li}^{2} \cdot$ Yecheng Deng ${ }^{1} \cdot$ Minglin $\mathrm{Ou}^{2}$
}

Received: 13 October 2021 / Accepted: 29 November 2021

Published online: 07 December 2021

(c) The Author(s) $2021 \quad$ OPEN

\begin{abstract}
Background Family with sequence similarity 65 member A (FAM65A), also known as RIPOR1, is differentially expressed between human tumor and non-tumor tissues in kinds of cancers. In addition, it was reported that the product of FAM65A may be a biomarker for cholangiocarcinoma patients. However, there is still no evidence on the relationship between the FAM65A and different types of tumors. Our study is mainly for exploring the prognostic values of FAM65A in pan-cancer and for further discovering a potential therapeutics target.

Methods We analyzed FAM65A expression, prognostic values, genetic alteration, protein phosphorylation, immune infiltration and enrichment analysis across different types of human malignant tumors based on The Cancer Genome Atlas (TCGA) and Gene Expression Omnibus (GEO) datasets. Additionally, Real-Time PCR (RT-qPCR) was performed to further confirm the roles of FAM65A in the pathogenesis of colorectal cancer.

Results We found that FAM65A expression was associated with the prognosis of multiple human tumors, especially colorectal cancer. Moreover, we also observed that FAM65A was highly expressed in colorectal cancer through RT-qPCR. We observed that decreasing phosphorylation level of the S351 locus in colon adenocarcinoma, uterine corpus endometrial carcinoma and lung adenocarcinoma. And the expression of FAM65A was positively related to cancer-associated fibroblasts (CAFs) infiltration in many tumors, such as colon adenocarcinoma. Therefore, FAM65A may be a potential prognostic biomarker of human tumors.
\end{abstract}

Keywords FAM65A · RIPOR1 · Prognostic biomarker · Pan-cancer ·TCGA · GEO

Wenken Liang and Chune Mo have contributed equally to this work.

Supplementary Information The online version contains supplementary material available at https://doi.org/10.1007/s12672-02100456-z.

Yecheng Deng, dyecheng@163.com; $\bowtie$ Minglin Ou, minglinou@163.com; minglinou@glmc.edu.cn|'College of Life Science, Guangxi Normal University, No. 1, Yanshan Middle Road, Guilin 541000, China. ${ }^{2}$ Central Laboratory, Guangxi Health Commission Key Laboratory of Glucose and Lipid Metabolism Disorders, The Second Affiliated Hospital of Guilin Medical University, No. 212, Renmin Road, Guilin 541000, China. ${ }^{3}$ Gastrointestinal Surgery, The Second Affiliated Hospital of Guilin Medical University, Guilin 541000, China. 


\section{Background}

There will be an estimated 18.1 million new cancer cases and 9.6 million cancer deaths in 2018, according to Global Cancer Statistics 2018 [1]. Tumors have gradually become the leading cause of death in the world [1]. With the continuous development of diagnosis and treatment technology, the survival time of cancer patients has been further improved. However, due to the complexity and stealthiness of the form and development of tumors, most patients don't receive timely treatment, and resulting in poor prognosis. Therefore, it is necessary that novel markers of early diagnosis are needed to apply in medical services. Pan-cancer analysis is valuable to reveal the correlation between any gene of interest and its clinical prognosis as well as its potential molecular mechanisms across different tumors [2]. In addition, pan-cancer analysis can also span the breadth of analyses and identify commonalities, differences, and emerging themes in human cancers [3]. TCGA Pan-Cancer analysis project, which is funded by USA National Institutes of health, includes functional genomics data in different tumors and provides the possibility for us to perform pan-cancer analysis [4-6].

It is now clear that RHO proteins are involved in almost every stage of tumorigenesis and some of the studies indicated that reduced RHO-protein function contributes to the morphological changes observed in tumor cells; this raises the dangerous possibility that inhibition of $\mathrm{RHO}$ proteins might promote a more aggressive tumor phenotype [7]. RHO family interacting cell polarization regulator 1 (RIPOR1) is also known as FAM65A, a novel Rho effector protein, which associates with GTP-bound RHO proteins (RHOA, RHOB, and RHOC) via an N-terminal HR1 domain [8]. It is noteworthy that FAM65A plays important roles in RHO-regulated the polarity of migrating cells via Golgi reorientation [8]. In addition, it is reported that FAM65A protein may be a biomarker for cholangiocarcinoma patients [9]. Therefore, we think that FAM65A may be one of the important genes for tumorigenesis and related to the prognosis of cancer patients. However, there is still no evidence on the association between FAM65A and different types of tumors based on the big clinical data.

In our study, we have comprehensively analyzed the correlation between the FAM65A expression and prognostic value as well as immune infiltration in different types of tumors. In addition, we also have conducted a series of analysis of FAM65A, including mutation, protein phosphorylation and gene enrichment, and so on.

\section{Materials and methods}

\subsection{Gene expression analysis}

The tumor immune estimation resource, TIMER2.0 web server (http://timer.cistrome.org/ accessed 25 July 2021) was used to explore FAM65A expression difference between primary tumor and the control based on the TCGA database [10]. For certain tumors without the expression information of normal tissue in the TCGA database, whose expression profile data was obtained through the GEPIA2 (http://gepia2.cancer-pku.cn/\#index accessed 25 July 2021) based on the GTEx database, under the setting of $\left|\log _{2} \mathrm{FC}\right|$ Cutoff $=1$, P-value cutoff $=0.01$ and "Match TCGA normal and GTEx data" [11]. To further confirm the expression difference of FAM65A between primary tumor and the control, the Oncomine web server (https://www.oncomine.org accessed 1 August 2021) was used to obtain the expression profile data.

In addition, we also tried to explore the association between FAM65A expression and the pathological stages of tumor through the GEPIA2 web server (accessed 25 July 2021). The violin plots indicate FAM65A expression in main stages (stage I, II, III, and IV) of different types of tumors.

UALCAN (http://ualcan.path.uab.edu/analysis-prot.html accessed 25 July 2021), an interactive web-portal for in-depth analysis of TCGA gene expression data, provides the possibility for us to conduct protein expression analysis of the CPTAC dataset $[12,13]$.

\subsection{The prognostic analysis of FAM65A}

The GEPIA2 web server (accessed 28 July 2021) was used to explore the FAM65A prognostic values in different types of tumors [11]. The significant survival maps of overall survival (OS) and disease-free survival (DFS) were obtained through the GEPIA2 web server, under setting of cutoff-high (50\%) and cutoff-low (50\%) values. Additionally, the Oncolnc web server (http://www.oncolnc.org/ accessed 16 August 2021) was also used to confirm the FAM65A prognostic values in colorectal cancer. Finally, the GSE17536 (probe name: 218029_at) cohort was downloaded to conduct statistical analysis 
from the PrognoScan (http://dna00.bio.kyutech.ac.jp/PrognoScan/index.html accessed 15 September 2021), in order to further confirm FAM65A in prognostic values of colon adenocarcinoma. We used the SPSS Statistics 23.0 to perform univariate and multivariable Cox analysis.

\subsection{Real-time PCR}

In this study, we have collected a few of human tissues from the Second Affiliated Hospital of Guilin Medical College in China, including colorectal cancer $(n=6)$ and non-tumor tissues $(n=4)$. All patients with colorectal cancer have signed an informed consent form. In addition, the ethics committee of the Second Affiliated Hospital of Guilin Medical College has approved the study. Total mRNA of samples was extracted using TRIzol (Invitrogen life technologies). Total mRNA was reverse transcribed to generate CDNA using the RT CDNA Synthesis kit. Quantitative polymerase chain reaction (qPCR) was performed using ViiA 7 Real-time PCR System (Applied Biosystems) and three replicates were performed for each sample.

The primer sequences were used:

\section{FAM65A}

Forward: 5'-GGCGAGTTTCATCTCCGAAT-3',

Reverse: 5'-AGACACTGCCCACAACCACA-3',

B-Actin

Forward: 5'-GTGGCCGAGGACTTTGATTG-3',

Reverse: 5'-CCTGTAACAACGCATCTCATATT-3'.

$2 \triangle \triangle C T$ method was used to quantify the relative $Z F C 3 H 1$ mRNA expression [14]. SPSS was used to perform statistical analyses ( $T$ test), and $P<0.05$ was deemed statistically significant.

a

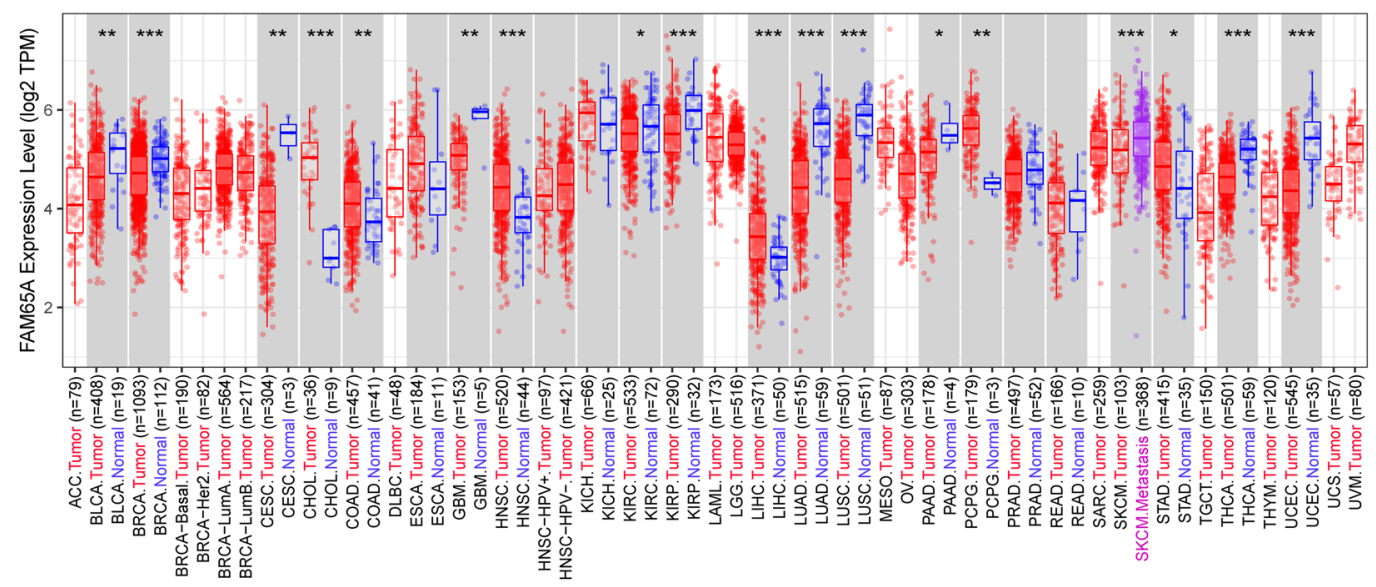

c

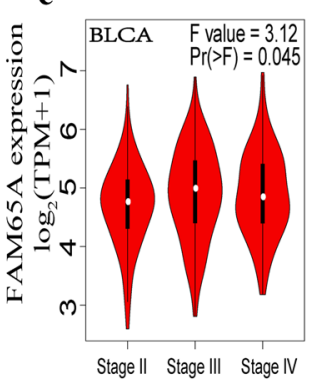

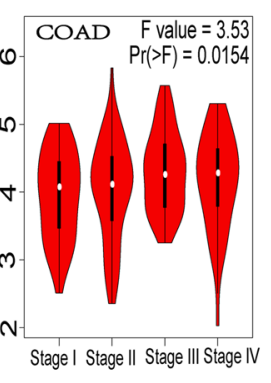

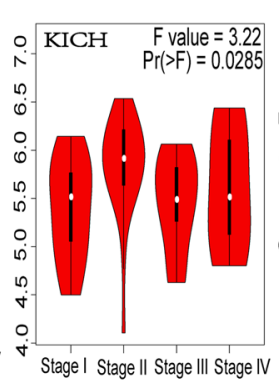

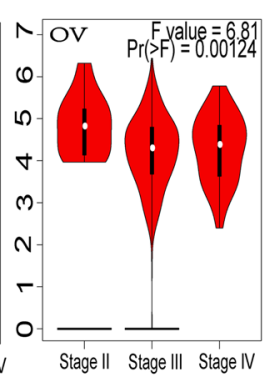

b

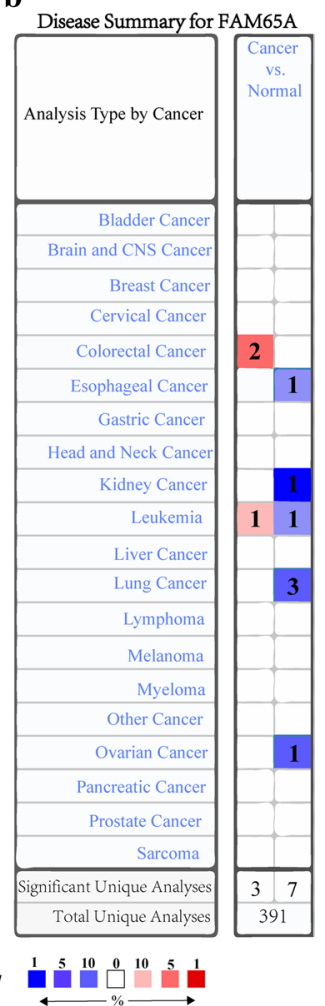

Fig. 1 The expression level of FAM65A gene in different tumors. a The expression levels of FAM65A in human tumors and corresponding normal tissues are obtained through the TIMER2. $\mathbf{b}$ The expression levels of FAM65A in human tumors and corresponding normal tissues are obtained through the oncomine. c The FAM65A expression violin plots in different pathological stages of BLCA, COAD, KICH, OV and THCA are obtained through the GEPIA2. ${ }^{*} \mathrm{P}<0.05,{ }^{* *} \mathrm{P}<0.01,{ }^{* * *} \mathrm{P}<0.001$ 


\subsection{FAM65A mutation in different types of tumors}

The cBioPortal web server (http://www.cbioportal.org accessed 1 August 2021) is a comprehensive website, which explore, visualize and analyze multidimensional cancer genomics data [15]. In this study, the genetic alteration feature of FAM65A was obtained through the "Cancer Types Summary" module of the cBioPortal web server (The gene name recognized by the system is RIPOR1). In addition, the correlation between FAM65A genetic alteration and survival prognosis (DFS, DSS, OS and PFS) was explored in the "Comparison" module. The prognostic values are presented with log-rank P values.

\subsection{Immune infiltration analysis}

We used the TIMER2.0 web server (accessed 18 August 2021) to evaluate the association between FAM65A expression and immune infiltration across different types of tumors. The result was analyzed through the Spearman's correlation test based on EPIC, MCPCOUNTED, XCELL and TIDE algorithms.

\subsection{Gene enrichment analysis}

The top 100 genes that are correlated with FAM65A were screened through "Similar Gene Detection" module of the GEPIA2 (accessed 22 August 2021). And the Pearson correlation between FAM65A and the top 5 genes is presented at the heatmap through the TIMER2.0 web server (accessed 26 August 2021). Furthermore, the Multi Experiment Matrix

$\mathbf{a}$

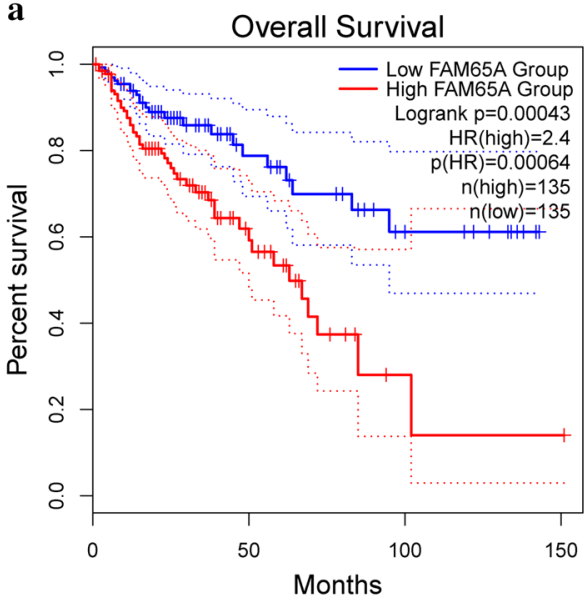

c Kaplan-Meier plot

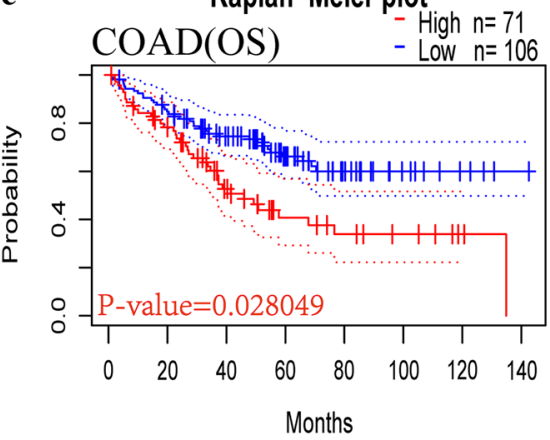

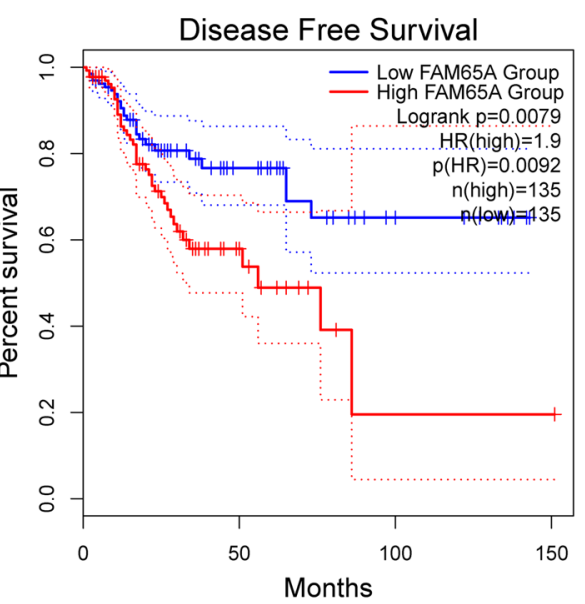

b

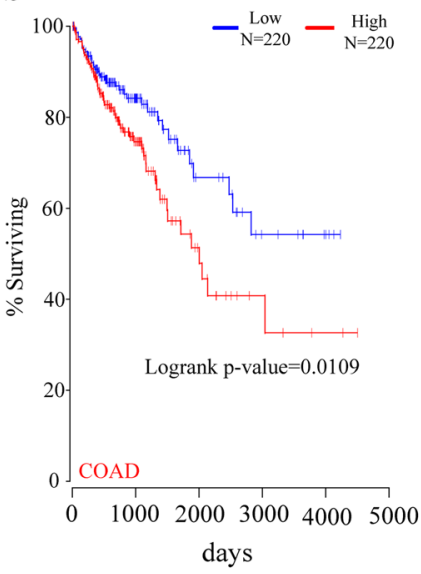

Kaplan-Meier plot

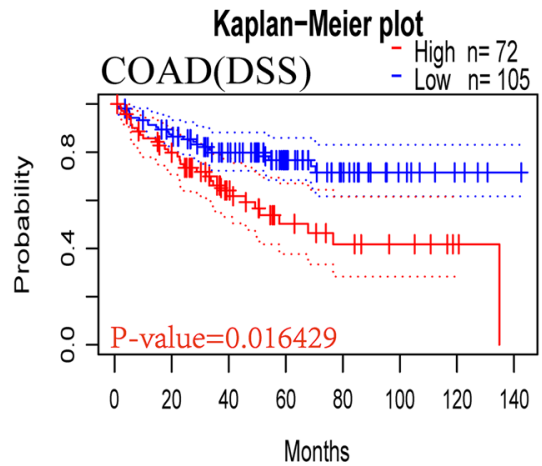

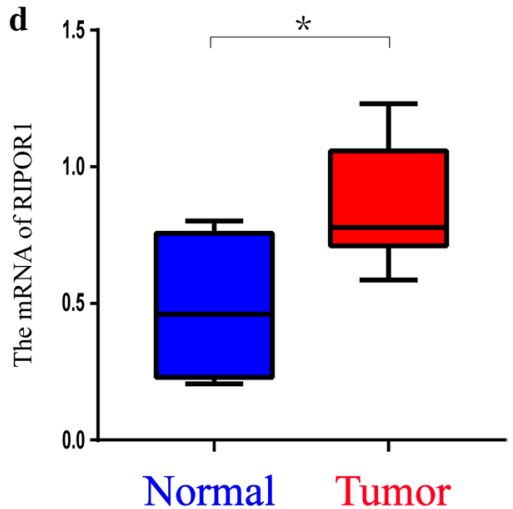

Fig. 2 Relationship between FAM65A gene expression and the prognostic values in colorectal cancer. a The FAM65A prognostic values were obtained through the GEPIA2 tool. b The FAM65A prognostic value was obtained through the Oncolnc. c The FAM65A prognostic values were obtained based on the GSE17536 cohort. d RT-qPCR 
Table 1 Univariate and multivariate cox analysis of clinical characteristics for COAD patients in GSE17536 cohort

\begin{tabular}{|c|c|c|c|c|c|c|c|c|}
\hline \multirow[t]{3}{*}{ Clinical variables } & \multicolumn{4}{|c|}{ GSE17536 (probe name:213065_at) } & \multicolumn{4}{|c|}{ GSE17536 (probe name:213065_at) } \\
\hline & \multicolumn{4}{|c|}{ Univariate analysis } & \multicolumn{4}{|c|}{ Multivariate analysis } \\
\hline & $\mathrm{HR}$ & HR.95L & HR.95H & $P$ value & $\mathrm{HR}$ & HR.95L & HR.95H & $P$ value \\
\hline Age & 0.997 & 0.977 & 1.017 & 0.746 & 1.010 & 0.986 & 1.035 & 0.407 \\
\hline Gender & 0.843 & 0.491 & 1.446 & 0.534 & 0.994 & 0.556 & 1.777 & 0.984 \\
\hline Differentiation & 2.133 & 1.241 & 3.665 & 0.006 & 1.569 & 0.864 & 2.850 & 0.139 \\
\hline Clinical stage & 3.623 & 2.495 & 5.262 & 0.000 & 3.923 & 2.607 & 5.903 & 0.000 \\
\hline FAM65A & 3.789 & 1.376 & 10.431 & 0.010 & 4.400 & 1.494 & 12.960 & 0.007 \\
\hline
\end{tabular}

Bold values indicate statistically significant factors that affect the prognosis of COAD patients

Differentiation Differentiation characteristics of cancer cells, $H R$ hazard ratio

(https://biit.cs.ut.ee/mem/index.cgi accessed 21 August 2021) was used to screen the top 50 genes that are co-expressed with FAM65A.

The Jvenn (http://bioinformatics.psb.ugent.be/webtools/Venn/ accessed 21 August 2021), an interactive Venn diagram viewer [16], which was used to compare an intersection between the co-expressed genes and the correlated genes. Moreover, two sets of genes that were combined to conduct KEGG pathway analysis through the DAVID tool (https:// david.ncifcrf.gov/ accessed 21 August 2021) and the "ggplot2" R packages.

At the same time, we used the Metascape web server (http://metascape.org/ accessed 26 August 2021) to perform $\mathrm{GO}$ enrichment analysis. After uploading the combined genes, "H sapiens” was selected to perform custom analysis, with
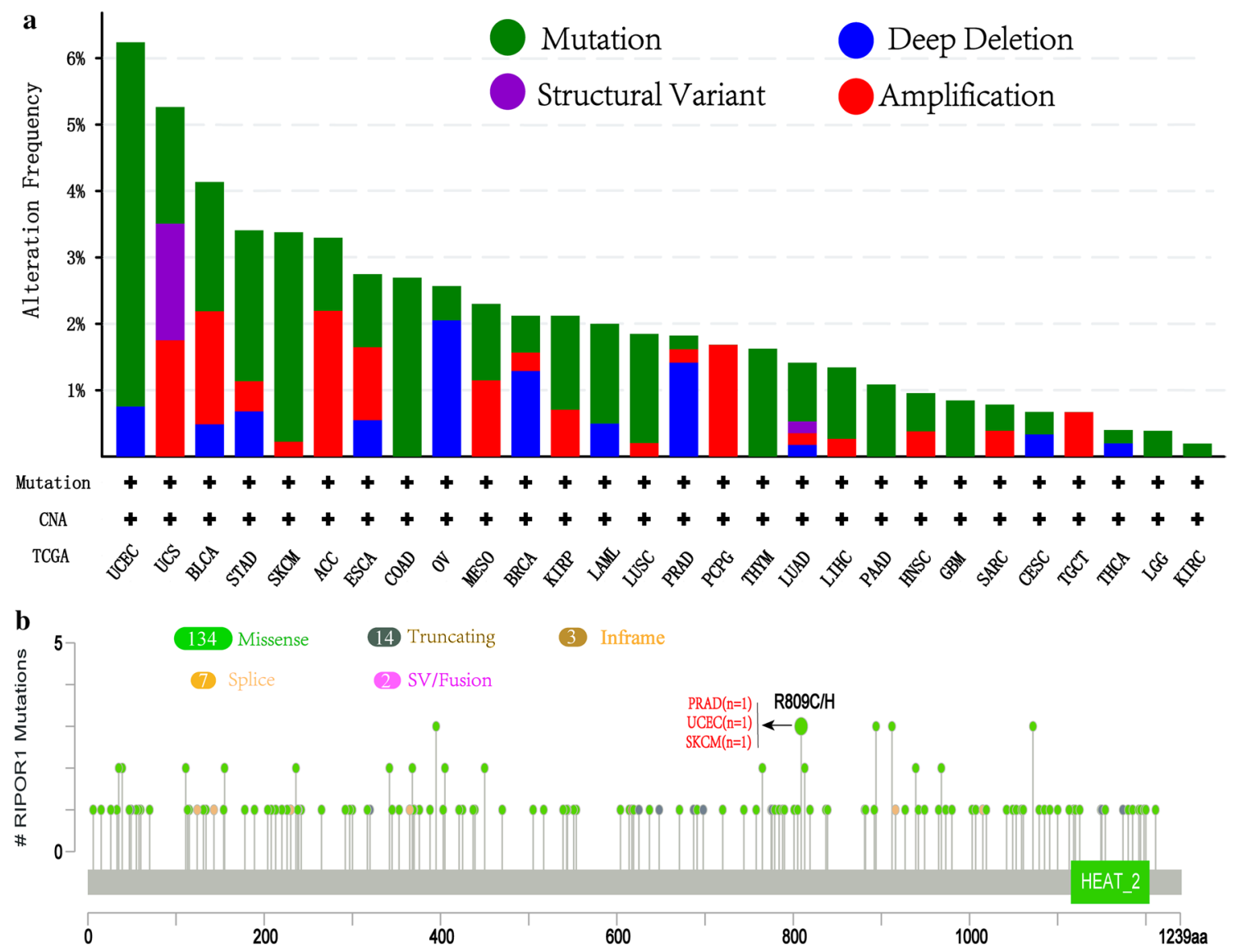

Fig. 3 Mutation feature of FAM65A in different tumors of TCGA dataset. The alteration frequency with mutation type (a) and mutation site (b) are showed 
the settings of Min Overlap $=3$, P-value Cutoff $=0.01$ and Min Enrichment $=1.5$. Finally, the STRING web server (https:// string-db.org/ accessed 26 August 2021) was used to screen the bound proteins with FAM65A. Entering the "FAM65A" for protein name, we selected "Homo sapiens" to obtain the PPI networks of FAM65A protein, with the main settings of network type ("full STRING network"), network edges ("evidence"), active interaction sources ("Experiments"), minimum required interaction score ["Iow confidence $(0.150)$ "], max number of interactors to show (1st shell: "no more than 50 interactors") and network display mode ("interactive svg").

\section{Results}

\subsection{Gene expression analysis}

Firstly, the TIMER2.0 was used to evaluate FAM65A expression difference between primary tumor and the control based on TCGA database. We found that FAM65A expression levels in tumor tissue of cholangio carcinoma (CHOL), head and neck squamous cell carcinoma (HNSC), hepatocellular carcinoma (LIHC), colon adenocarcinoma (COAD), pheochromocytoma and paraganglioma (PCPG) and stomach adenocarcinoma (STAD) are higher than the corresponding normal tissue based on TCGA dataset (Fig. 1a). In contrary, FAM65A expression levels in tumor tissue of breast invasive carcinoma (BRCA), kidney renal papillary cell carcinoma (KIRP), lung adenocarcinoma (LUAD), lung squamous cell carcinoma (LUSC), uterine corpus endometrial carcinoma (UCEC), cervical squamous cell carcinoma and endocervical adenocarcinoma (CESC), Glioblastoma multiforme (GBM), kidney renal clear cell carcinoma (KIRC) and Pancreatic adenocarcinoma (PAAD) are lower than the corresponding normal tissue based on TCGA dataset (Fig. 1a). Moreover, the normal tissues in the GTEx dataset serve as the controls and the results are presented in Fig. S1.

At the same time, the Oncomine web server was used to further confirm the expression difference between primary tumor and the control. The suppressed expression level of FAM65A was observed in esophageal cancer, kidney cancer, lung cancer and ovarian cancer, while elevated expression level of FAM65A was observed in colorectal cancer, compared with the control (Fig. 1b). In addition, the UALCAN tool was used to evaluate the FAM65A total protein in different types of tumors based on the CPTAC dataset. And the results are presented in Fig. S1. Finally, we used the GEPIA2 to observe the association between FAM65A expression and different pathological stages of tumor, such as colon adenocarcinoma (Fig. 1c).

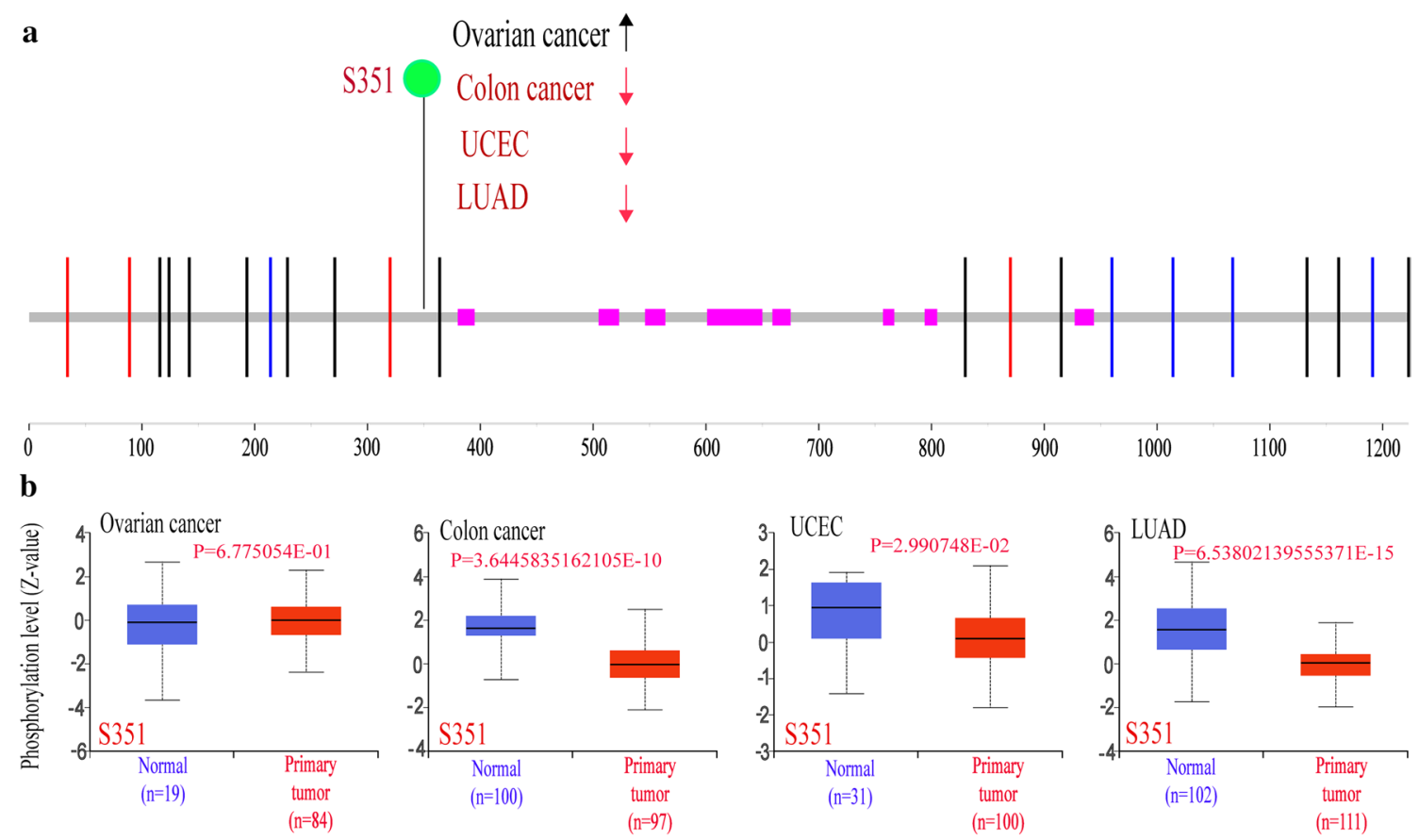

Fig. 4 Phosphorylation analysis of RIPOR1 protein in different tumors. a The phosphoprotein site (S351) is showed in the schematic diagram of the RIPOR1 protein. $\mathbf{b}$ The box plots of different tumors, including ovarian cancer, colon cancer, UCEC and LUAD 
a

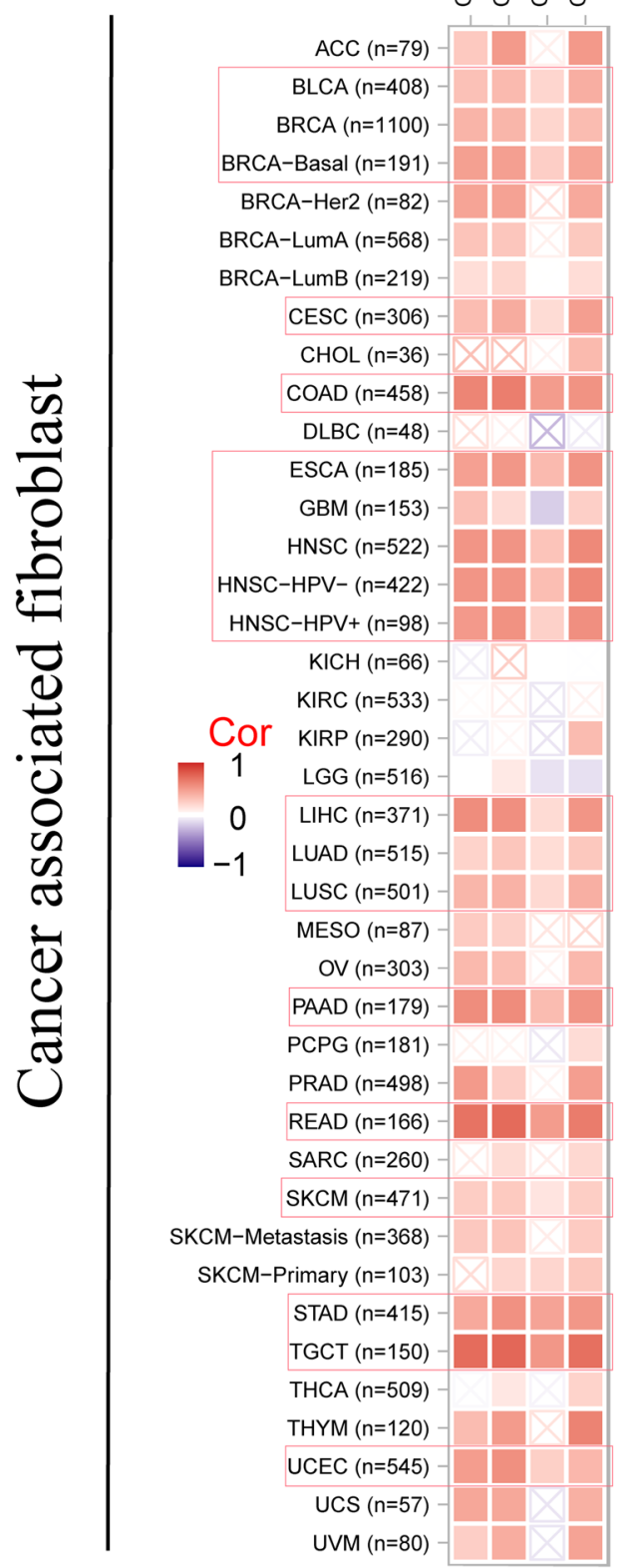

b
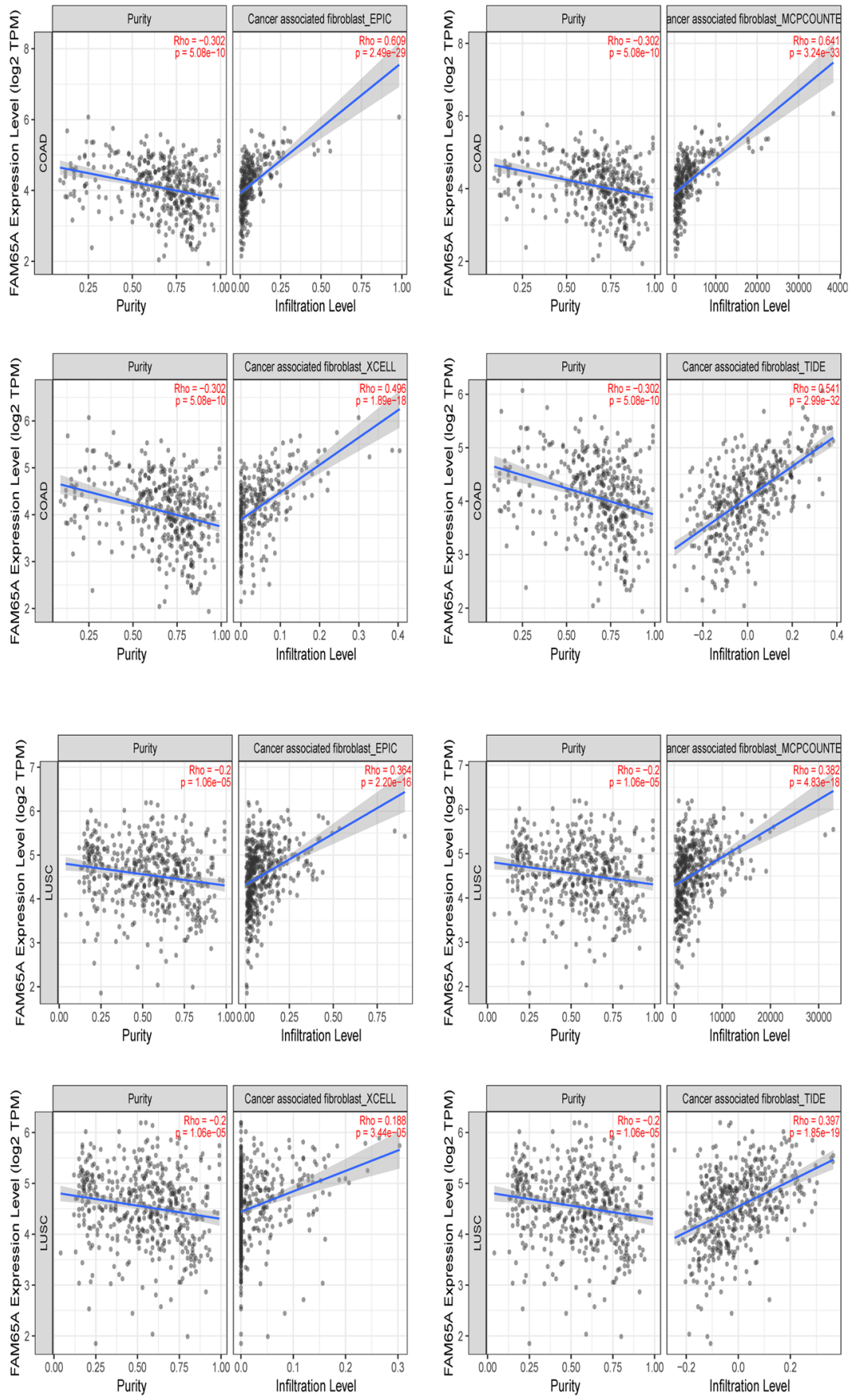

Fig. 5 Correlation analysis between FAM65A expression and immune infiltration. a The correlation between FAM65A expression and the immune infiltration of CAFs in different tumors is presented based on EPIC, MCPCOUNTED, XCELL and TIDE algorithms. $\mathbf{b}$ The scatter plot data of COAD and LUSC are presented 
Fig. 6 FAM65A-related gene enrichment analysis. a The top 100 FAM65A-related genes are obtained through the GEPIA2 tool based on TCGA database and the expression correlation between FAM65A and SLC39A13, SLC12A4, PSKH1, PLA2G15 and C16ORF86 is shown. b The intersection between FAM65A correlated and co-expression genes was presented in a Venn diagram, which showed four common members, namely SORBS3, SYDE1, ATP6V0D1 and CYB5R3. c KEGG pathway analysis was conducted. d The FAM65A-binding proteins confirmed by the experimental methods are presented, using the STRING tool

\subsection{The prognostic analysis of FAM65A}

We used the GEPIA tool to investigate the correlation between FAM65A expression and the survival prognosis of patients across different types of tumors. And the relationship between FAM65A expression and the prognostic values of pancancer is presented in Fig. S2. In this study, we focused on colorectal cancer and the highly expressed FAM65A was linked to poor overall survival and disease-free survival in the COAD patients (Fig. 2a). At the same time, the Oncolnc web server was used to further confirm the prognostic value of COAD and the result is presented in Fig. $2 \mathrm{~b}$.

In addition, the GSE17536 (probe name: 218029_at) cohort was downloaded to perform statistical analysis. COAD patients with high FAM65A expression had shorter overall survival and disease-specific survival in the GSE17536 cohort (Fig. 2c). Additionally, cox univariable analysis indicated clinical stage ( $H R=3.623, P<0.001)$, cell differentiation $(\mathrm{HR}=2.133, \mathrm{P}=0.006)$ and $F A M 65 A$ expression $(\mathrm{HR}=3.789, \mathrm{P}=0.010)$ influenced the prognosis of COAD patients. However, multivariable cox analysis suggested $F A M 65 A$ expression $(H R=4.400, P=0.007)$ wasn't an independent factor to influence COAD prognosis (Table 1).

Finally, RT-qPCR were conducted to explore the expression difference between tumor and non-tumor tissues of colorectal cancer. As shown in Fig. 2d, we observed that the FAM65A relative expression level of tumors were higher than non-tumor tissues in colorectal cancer $(P<0.05)$, which was similar to the result observed by the bioinformatics analysis.

\subsection{FAM65A mutation in different types of tumors}

The genetic alteration feature of FAM65A in kinds of tumors was explored based on the TCGA cohorts. As shown in Fig. 3a, we found that the patients with UCEC had the highest alteration frequency of FAM65A (> 6\%) with "mutation" as the main type. "Mutation" as the unique form of genetic alteration existed in all patients with COAD, thymoma (THYM), PAAD, GBM, brain lower grade glioma (LGG) and KIRC. The sites, types and case number of FAM65A genetic alteration are presented in Fig. 3b.

We observed that the primary type of $F A M 65 A$ genetic alteration was missense mutation. $\mathrm{R} 809 \mathrm{C} / \mathrm{H}$ alteration, which was found in one case of PRAD, one case of UCEC and one case of SKCM, leads to a frame shift mutation of FAM65A, translation from $\mathrm{R}$ (Arginine) to $\mathrm{C}$ (Cysteine) or $\mathrm{H}$ (Histidine) at the 809 site of FAM65A protein and FAM65A protein was subsequently truncated. In addition, the potential correlation between FAM65A genetic alteration and the prognosis with UCEC, prostate adenocarcinoma (PRAD) and skin cutaneous melanoma (SKCM) is presented in Fig. S3.

\subsection{FAM65A protein phosphorylation}

The difference of FAM65A phosphorylation level in different tumors was explored based on the CPTAC dataset. Figure 4 indicates that the S351 locus presents a higher phosphorylation level for the control of COAD, UCEC and LUAD (Fig. 4a, b), but not ovarian cancer $(P=6.7 \mathrm{e}-1)$. Moreover, the potential relationship between phosphorylation and the survival prognosis of colorectal cancer was explored. However, we found no significant difference (Fig. S4).

\subsection{Immune infiltration analysis}

In this study, the potential association between FAM65A expression and infiltration level of CAFs was investigated based on different algorithms (EPIC, MCPCOUNTED, XCELL and TIDE). We observed that a statistically positive relationship between FAM65A expression and the CAFs infiltration for most tumors (Fig. 5a). Additionally, the scatter plots of COAD and LUSC are presented in Fig. $5 b$. 
$\mathbf{a}$

$\begin{array}{rr}\mathrm{P}<0.05 & 1 \\ \boldsymbol{\mathrm { P }}>0.05 & 0 \\ -1\end{array}$

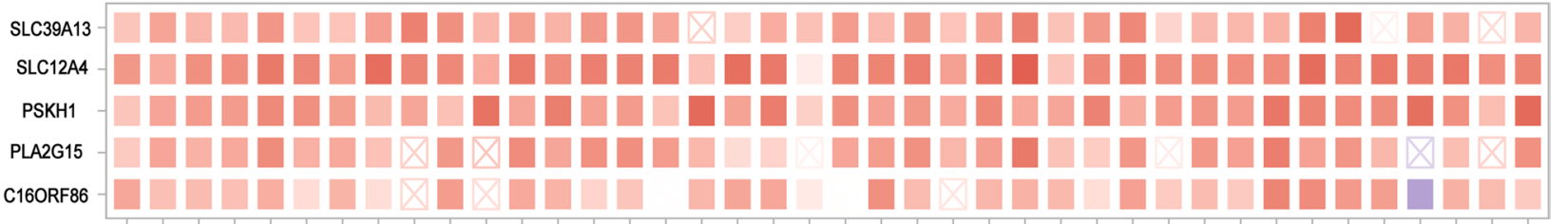

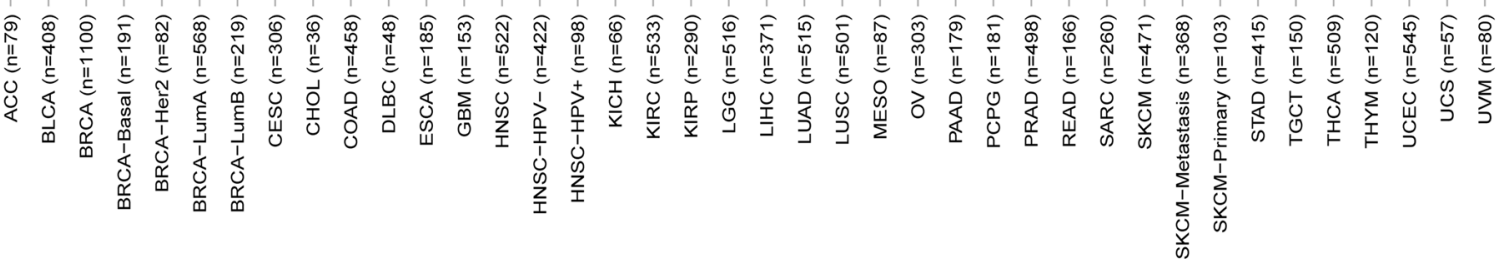

b

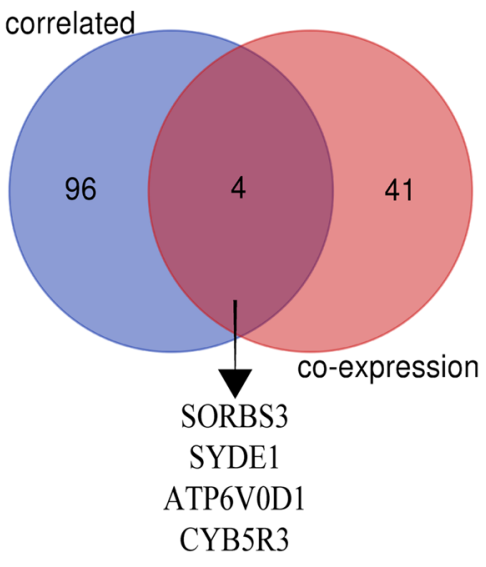

c

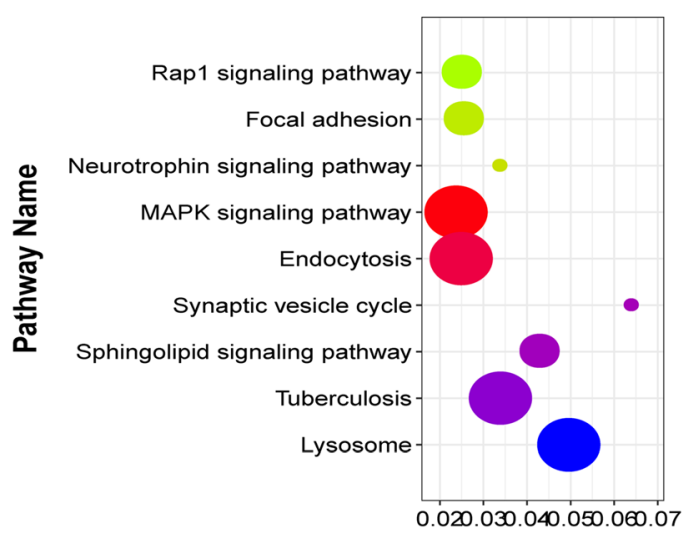

KEGG Enrichment Scatter Plot

Gene Number

$\begin{array}{r}-4.0 \\ \hline\end{array}$

5.0

5.5

6.0

p Value

0.0475

0.0243

0.0011

Rich Factor

d

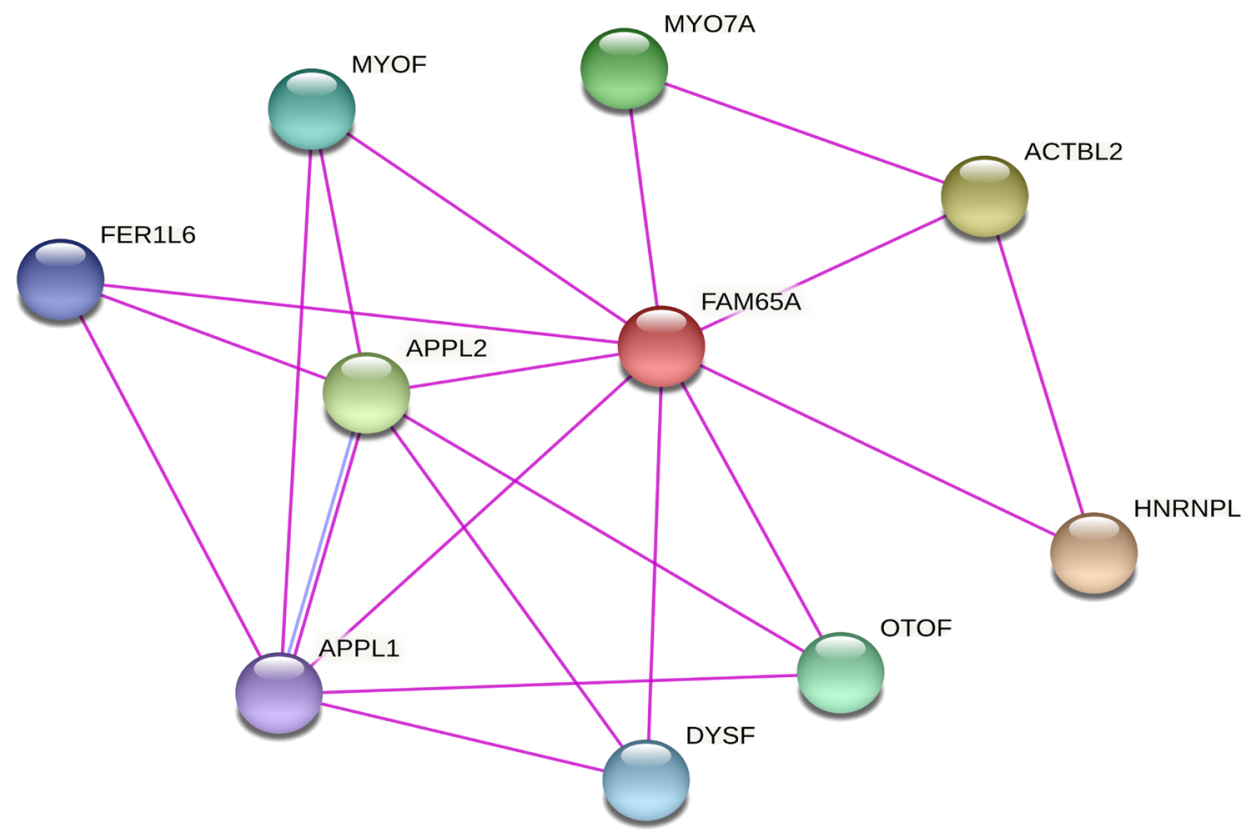

Experimentally Determined 


\subsection{Gene enrichment analysis}

We tried to screen FAM65A correlated and co-expression genes to perform a series of pathway enrichment analysis in order to further explore the FAM65A potential mechanisms in human tumors. There is a positive correlation between FAM65A and the five genes (SLC39A13, SLC12A4, PSKH1, PLA2G15 and C16ORF86) in most tumors, and the relationship is presented in the heatmap (Fig. 6a). Moreover, the intersection between FAM65A correlated and co-expression genes is presented in a Venn diagram, which showed four common members, namely SORBS3, SYDE1, ATP6V0D1 and CYB5R3 (Fig. 6b).

Then, we combined the above genes to conduct KEGG and GO enrichment analyses. KEGG analysis suggested that the combined genes were mainly enriched in "lysosome", "endocytosis" and "MAPK signalling pathway", which might participate in the effect of FAM65A on tumorigenesis (Fig. 6c). Additionally, GO enrichment analysis indicated most of genes are related to cell physiological activity, such as "regulation of cellular protein localization", "endosome membrane" and "protein domain specific binding", and so on (Fig. S5). Finally, the PPI network of FAM65A described by STRING to explore the interacting protein showed 11 nodes, 17 edges, and the PPI enrichment p-value 0.0483 (Fig. 6d).

\section{Discussion}

Pan-cancer analysis is critical and helpful for comparing the similarities and differences between different malignant tumors, which contribute to provide novel insights into tumor biomarkers and cancer prevention. In recent years, many studies have provided a new understanding for whole-genome analysis of human pan-cancer and suggested the close relationship between tumorigenesis and driver genes, mutations, copy number alterations, and tumor purity, which is critical to the diagnosis and treatment of tumors [17-21].

The Rho-family control pathways involved in cellular morphology, which are commonly activated in cancer cell invasion and metastasis [22]. And Rho guanosine triphosphatases (GTPases) have well-recognized roles in regulating various downstream signaling pathways in a wide range of cancers [23]. RHO family interacting cell polarization regulator 1 (RIPOR1) is also known as FAM65A, a novel Rho effector protein, which associates with GTP-bound RHO proteins (RHOA, $\mathrm{RHOB}$, and $\mathrm{RHOC}$ ) via an N-terminal HR1 domain, plays an important role in RHO-regulated the polarity of migrating cells via Golgi reorientation [8]. In addition, it is reported that FAM65A protein may be a biomarker for cholangiocarcinoma patients [9]. However, the roles of FAM65A in human pan-cancer are still unknown. In this study, we explored the correlation of FAM65A in different cancer tumorigenesis models by a pan-cancer analysis based on multiple databases. In this study, we first revealed that the FAM65A expression is abnormal at mRNA level in most human tumors. This indicates that FAM65A may serve as an oncogene in different common cancers. According to KM survival curves, including OS, DFS and DSS, FAM65A was confirmed as a potential biomarker of pan-cancer.

Gene mutations play an important role in the pathogenesis of some human tumors [24]. In this study, we observed that the "mutation" was the primary form of DNA alterations of FAM65A in all tumors based on the TCGA cohort. It is noteworthy that specific gene mutation is valuable to predict the prognosis of some tumor patients. Phosphorylation is an essential regulatory mechanism in several proteins [25]. The mammalian target of rapamycin (mTOR) is a key regulator of cell growth and proliferation [26]. As early as 2009, the S351 locus phosphorylation of FAM65A was experimentally confirmed in PubMed ID 19276368 [27]. Our findings showed that the expression level of FAM65A total protein phosphorylation at the $\mathrm{S} 351$ site was down-regulated in the primary tumors.

A report have shown that cancer-associated fibroblasts (CAFs) play an important role in tumor progression, including carcinogenesis, invasion, metastasis and the chemoresistance of cancer cells [28]. As one of the hallmarks of cancer, development of metastasis accounts for more than $90 \%$ cancer-related deaths [29]. It is noteworthy that CAFs secreted exosomes promote metastasis and chemotherapy resistance of colorectal cancer [30, 31]. In this study, we found that FAM65A expression was positively associated with CAFs infiltration in colorectal cancer. Therefore, we concluded that the colorectal cancer with high FAM65A expression may promote the infiltration level of CAFs, which lead to a poor survival prognosis for the patients.

MAPK cascades are central signalling elements that regulate basic processes including cell proliferation, differentiation and stress responses [32-34]. MAPK signaling is one of the best-defined pathways in cancer biology, and its hyperactivation is responsible for over $40 \%$ human cancer cases [35]. In this study, we integrated FAM65A correlated 
and co-expression genes to perform KEGG and GO enrichment analyses, and identified the potential impact of MAPK signalling pathway and MAPK cascade in the pathogenesis of human tumors.

\title{
5 Conclusions
}

In summary, our findings contribute to further explore the roles of FAM65A in tumorigenesis. Above all, we first indicted that FAM65A may be a novel prognostic biomarker of pan-cancer, especially colorectal cancer.

\begin{abstract}
Authors' contributions Conception and design: WL, YD, MO, CM; collection and assembly of data:WG, WC, WL, JW, JS, XH, YD, MO, JW; development of methodology: WC, WL, YD, MO; data analysis and interpretation: WC, WG, WL, YD, MO, JW; manuscript drafting: WL, MO; manuscript revision: WC, WG, YD, MO. All authors read and approved the final manuscript.
\end{abstract}

Funding This work was supported by the Guangxi Natural Science Foundation (No. 2020GXNSFAA159124, MO), the National Natural Science Foundation of China (No. 82060393, MO).

Data availability The raw data are available at GEO: GSE17536.

Code availability $R$ 3.5.0, SPSS 23 and Cytoscape 3.8.2.

\section{Declarations}

Ethics approval and consent to participate Our study involves human tissues derived from tumor tissues and normal tissues of colorectal patients. All patients signed an informed consent form, and the ethics committee of the Second Affiliated Hospital of Guilin Medical College has approved the study.

Competing interests The authors declare that they no competing interests.

Open Access This article is licensed under a Creative Commons Attribution 4.0 International License, which permits use, sharing, adaptation, distribution and reproduction in any medium or format, as long as you give appropriate credit to the original author(s) and the source, provide a link to the Creative Commons licence, and indicate if changes were made. The images or other third party material in this article are included in the article's Creative Commons licence, unless indicated otherwise in a credit line to the material. If material is not included in the article's Creative Commons licence and your intended use is not permitted by statutory regulation or exceeds the permitted use, you will need to obtain permission directly from the copyright holder. To view a copy of this licence, visit http://creativecommons.org/licenses/by/4.0/.

\section{References}

1. Bray F, et al. Global cancer statistics 2018: GLOBOCAN estimates of incidence and mortality worldwide for 36 cancers in 185 countries. CA Cancer J Clin. 2018;68(6):394-424.

2. Chen $Y$, et al. Pan-cancer analysis reveals an immunological role and prognostic potential of PXN in human cancer. Aging. 2021;13(12):16248-66.

3. Cui K, et al. Comprehensive characterization of the rRNA metabolism-related genes in human cancer. Oncogene. 2020;39(4):786-800.

4. Blum A, Wang P, Zenklusen JC. SnapShot:TCGA-analyzed tumors. Cell. 2018;173(2):530.

5. Tomczak K, Czerwińska P, Wiznerowicz M. The Cancer Genome Atlas (TCGA): an immeasurable source of knowledge. Contemp Oncol. 2015;19(1a):A68-77.

6. Clough E, Barrett T. The gene expression omnibus database. Methods Mol Biol. 2016;1418:93-110.

7. Sahai E, Marshall CJ. RHO-GTPases and cancer. Nat Rev Cancer. 2002;2(2):133-42.

8. Mardakheh FK, Self A, Marshall CJ. RHO binding to FAM65A regulates Golgi reorientation during cell migration. J Cell Sci. 2016;129(24):4466-79.

9. Kotawong K, et al. Plasma phosphoproteome and differential plasma phosphoproteins with Opisthorchis viverrini-related cholangiocarcinoma. Asian Pac J Cancer Prev. 2015;16(3):1011-8.

10. Li T, et al. TIMER: a web server for comprehensive analysis of tumor-infiltrating immune cells. Cancer Res. 2017;77(21):e108-10.

11. Tang Z, et al. GEPIA2: an enhanced web server for large-scale expression profiling and interactive analysis. Nucleic Acids Res. 2019;47(W1):W556-60.

12. Chandrashekar DS, et al. UALCAN: a portal for facilitating tumor subgroup gene expression and survival analyses. Neoplasia. 2017;19(8):649-58.

13. Chen F, et al. Pan-cancer molecular subtypes revealed by mass-spectrometry-based proteomic characterization of more than 500 human cancers. Nat Commun. 2019;10(1):5679.

14. Arab A, et al. Potential circulating miRNA signature for early detection of NSCLC. Cancer Genet. 2017;216-217:150-8.

15. Gao J, et al. Integrative analysis of complex cancer genomics and clinical profiles using the cBioPortal. Sci Signal. 2013;6(269):pl1. 
16. Bardou P, et al. jvenn: an interactive Venn diagram viewer. BMC Bioinform. 2014;15:293.

17. Zack TI, et al. Pan-cancer patterns of somatic copy number alteration. Nat Genet. 2013;45(10):1134-40.

18. Aran D, Sirota M, Butte AJ. Systematic pan-cancer analysis of tumour purity. Nat Commun. 2015;6:8971.

19. Ma X, et al. Pan-cancer genome and transcriptome analyses of 1,699 paediatric leukaemias and solid tumours. Nature. 2018;555(7696):371-6.

20. Saghafinia S, et al. Pan-cancer landscape of aberrant DNA methylation across human tumors. Cell Rep. 2018;25(4):1066-1080.e8.

21. Priestley $P$, et al. Pan-cancer whole-genome analyses of metastatic solid tumours. Nature. 2019;575(7781):210-6.

22. Corry J, Mott HR, Owen D. Activation of STAT transcription factors by the Rho-family GTPases. Biochem Soc Trans. 2020;48(5):2213-27.

23. Zeng RJ, et al. Rho GTPases in cancer radiotherapy and metastasis. Cancer Metastasis Rev. 2020;39(4):1245-62.

24. Martínez-Jiménez F, et al. A compendium of mutational cancer driver genes. Nat Rev Cancer. 2020;20(10):555-72.

25. Beausoleil SA, et al. Large-scale characterization of HeLa cell nuclear phosphoproteins. Proc Natl Acad Sci USA. 2004;101(33):12130-5.

26. Azim H, Azim HA, Escudier B. Targeting mTOR in cancer: renal cell is just a beginning. Target Oncol. 2010;5(4):269-80.

27. Chen RQ, et al. CDC25B mediates rapamycin-induced oncogenic responses in cancer cells. Cancer Res. 2009;69(6):2663-8.

28. An Y, et al. Crosstalk between cancer-associated fibroblasts and immune cells in cancer. J Cell Mol Med. 2020;24(1):13-24.

29. Seyfried TN, Huysentruyt LC. On the origin of cancer metastasis. Crit Rev Oncog. 2013;18(1-2):43-73.

30. Hu JL, et al. CAFs secreted exosomes promote metastasis and chemotherapy resistance by enhancing cell stemness and epithelial-mesenchymal transition in colorectal cancer. Mol Cancer. 2019;18(1):91.

31. Ren J, et al. Carcinoma-associated fibroblasts promote the stemness and chemoresistance of colorectal cancer by transferring exosomal IncRNA H19. Theranostics. 2018;8(14):3932-48.

32. Keshet $Y$, Seger R. The MAP kinase signaling cascades: a system of hundreds of components regulates a diverse array of physiological functions. Methods Mol Biol. 2010;661:3-38.

33. Sabio G, Davis RJ. TNF and MAP kinase signalling pathways. Semin Immunol. 2014;26(3):237-45.

34. Plotnikov A, et al. The MAPK cascades: signaling components, nuclear roles and mechanisms of nuclear translocation. Biochim Biophys Acta. 2011;1813(9):1619-33.

35. Yuan J, et al. The MAPK and AMPK signalings: interplay and implication in targeted cancer therapy. J Hematol Oncol. $2020 ; 13(1): 113$.

Publisher's Note Springer Nature remains neutral with regard to jurisdictional claims in published maps and institutional affiliations. 